arch-ive/9506037

\title{
Crystal surfaces with correlated disorder: Phase transitions between roughening and superroughening
}

\author{
Stefan Scheidl \\ Institut für Theoretische Physik, Universität zu Köln, Zülpicher Str. 77, D-50937 Köln, Germany
}

(May 30, 1995)

\begin{abstract}
A theory for surface transitions in the presence of a disordered pinning potential is presented. Arbitrary disorder correlations are treated in the framework of a dynamical functional renormalization group. The roughening transition, where surface roughness and mobility behave discontinuously, is shown to turn smoothly into the continuous superroughening transition, when the range of disorder correlations is decreased. Implications for random-field $X Y$-models and vortex glasses are discussed.
\end{abstract}

PACS: 64.40, 64.70.Pf, 68.35.Rh, 75.60.Ge

Typeset using REVTEX 
The shape of a fluctuating crystal surface is affected by the pinning potential provided by the crystal planes. The interface exhibits a roughening transition, if these planes are perfect, and it exhibits a superroughening transition, when these planes are strongly disordered. The roughening transition is well understood theoretically and verified in different experimental situations (see e.g. [1]2] and references therein). Above the roughening temperature $T_{\mathrm{R}}$ the interface is thermally rough, whereas below $T_{\mathrm{R}}$ the interface becomes smooth, since it locks into the pinning potential. However, for quenched bulk disorder [3] and substrate disorder [4.5] it was shown, that the transition has a different nature. This so-called superroughening transition occurs at $T_{\mathrm{SR}}=T_{\mathrm{R}} / 2$. Above $T_{\mathrm{SR}}$ the interface is thermally rough again, but below $T_{\mathrm{SR}}$ the disordered pinning potential increases the roughness of the interface. In this Letter we address the question: how are the roughening transition and the superroughening transition related? What happens "in-between", if we switch from a pinning potential with long-range correlations to short-range correlations?

As shown below, new physics emerges for logarithmic substrate roughness. This can be realized, if the crystal is grown on a logarithmically rough substrate. Such a substrate could be generated by quenching it from a temperature above its roughening transition. In order to have a stable substrate surface, the roughening temperature of the substrate should be higher than that of the crystal.

Our analysis is also relevant for a $X Y$-model in the presence of a random field, as long as vortices can be neglected [6]. Different types of sources of the random field may have different multipolar character, which lead to a power-law decay of correlations.

Finally, the consideration applies to two-dimensional vortex glasses [7],8]. Their pinning can be caused by local suppressions of the condensate density, which also relaxes with power laws and leads in turn to a power-law decay of correlations. This power varies with the dimensionality of the defects.

At present there is a controversial discussion about the actual roughness of the superrough phase. Renormalization group (RG) calculations [4.5], variational calculations [9] and simulations [10] give an inconsistent picture. In view of this controversy we focus on topics 
consistently described by these different approaches: the phase boundary and the nature of the phase transition. The present study is based on a functional dynamical renormalization group applying to the limit of very weak pinning.

The interface profile is described by $\phi$ as a function of time $t$ and the two-dimensional lateral coordinate $\mathbf{r}$. Its kinetics is captured by the overdamped equation of motion

$$
\begin{aligned}
m^{-1} \partial_{t} \phi(t, \mathbf{r})= & K \nabla^{2} \phi(t, \mathbf{r})-\chi \sin [\phi(t, \mathbf{r})-d(\mathbf{r})]+ \\
& +F+\xi(t, \mathbf{r})
\end{aligned}
$$

Here $m$ is a mobility, $K$ is the surface stiffness, $\chi$ is the amplitude of the periodic pinning potential, which has a quenched disorder phase $d$ representing deformed crystal planes, $F$ is a driving force per unit area, and $\xi$ is thermal noise at temperature $T$. We implicitly suppose a cutoff $\Lambda$ for the wave-vector of shape fluctuations, which corresponds to an area $4 \pi / \Lambda^{2}$ per degree of freedom in the lateral plane. Since we assume regularization in momentum space, we consider $\phi$ and $d$ to be defined continuously on the whole lateral plane (see Fig. 1) and not just only on a lattice of points $\mathbf{r}$ [1].

The disorder field is characterized by zero mean and a difference correlation $\overline{\left[d(\mathbf{r})-d\left(\mathbf{r}^{\prime}\right)\right]^{2}}=2 \Delta\left(\mathbf{r}-\mathbf{r}^{\prime}\right)$, which for simplicity is taken to be isotropic. For the following it is convenient to introduce the correlation $\left(g_{0}:=\frac{1}{2} m^{2} \chi^{2}\right)$

$$
\gamma_{0}(\mathbf{r}):=g_{0} \overline{e^{i[d(\mathbf{r})-d(\mathbf{0})]}}=g_{0} e^{-\Delta(\mathbf{r})}
$$

In terms of this function, a perfect crystal exhibiting the roughening transition has $\gamma_{0}(\mathbf{r})=g$. In the studies of the superroughening transition [3 [5], $\gamma_{0}(\mathbf{r})$ was supposed to decay rapidly for large r. Eq. (2) shows, that this case corresponds to a very rough substrate with a rapidly increasing function $\Delta(\mathbf{r})$. Here we allow for a general, possibly slowly decaying correlation $\gamma_{0}(\mathbf{r})$

In constructing the RG, we choose an approach different from previous treatments. Since we do not want to specialize to a special correlation, we have to perform a functional RG. This can be achieved in the formalism of Martin, Siggia, and Rose [12], which requires the 
introduction of an additional field $\tilde{\phi}$. In this formulation the disorder average can easily be performed. The generating functional then reads

$$
Z=\int \mathcal{D} \tilde{\phi} \mathcal{D} \phi \exp \left[\mathcal{A}_{0}\right]
$$

with the action $\mathcal{A}_{0}=\mathcal{A}_{0}^{(0)}+\mathcal{A}_{0}^{(d)}$ composed of free part and disorder part

$$
\begin{aligned}
\mathcal{A}_{0}^{(0)}= & \int d t d^{2} r\left\{\tilde{J}_{0}(t, \mathbf{r}) \tilde{\phi}_{0}(t, \mathbf{r})+\frac{1}{2} \vartheta_{0} \tilde{\phi}_{0}^{2}(t, \mathbf{r})-\right. \\
& \left.-\tilde{\phi}_{0}(t, \mathbf{r})\left[\mu_{0}^{-1} \dot{\phi}_{0}(t, \mathbf{r})-\kappa_{0} \nabla^{2} \phi_{0}(t, \mathbf{r})\right]\right\} \\
\mathcal{A}_{0}^{(d)}= & \int d t d t^{\prime} d^{2} r d^{2} r^{\prime} \frac{1}{2} \gamma_{0}\left(\mathbf{r}-\mathbf{r}^{\prime}\right) \times \\
& \times \tilde{\phi}_{0}(t, \mathbf{r}) \tilde{\phi}_{0}\left(t^{\prime}, \mathbf{r}^{\prime}\right) \cos \left[\phi_{0}(t, \mathbf{r})-\phi_{0}\left(t^{\prime}, \mathbf{r}^{\prime}\right)\right]
\end{aligned}
$$

We introduced the unrenormalized quantities $\phi_{0}=\phi, \vartheta_{0}=2 m T, \mu_{0}=1, \kappa_{0}=m K$, and $\tilde{J}_{0}=m F$.

In the absence of the pinning potential $\left(\chi=0\right.$ and $\left.\gamma_{0}=0\right)$ one has correlations

$$
\begin{aligned}
C^{(0)}(t, \mathbf{r}) & :=\frac{1}{2}\left\langle[\phi(t, \mathbf{r})-\phi(0, \mathbf{0})]^{2}\right\rangle^{(0)} \\
& =n \hat{C}^{(0)}\left(\mu \kappa \Lambda^{2} t, \Lambda r\right) \\
\hat{C}^{(0)}(\tau, \rho) & =2 \int_{0}^{1} \frac{d x}{x}\left[1-\mathrm{J}_{0}(x \rho) e^{\left.-x^{2}|\tau|\right]}\right. \\
& \approx \ln \max \left(1,|\tau|, \rho^{2}\right) .
\end{aligned}
$$

$\mathrm{J}_{0}$ denotes the Bessel function and $n:=\mu \vartheta /(8 \pi \kappa)=T /(4 \pi K)$ is the roughness coefficient of the unpinned interface.

We perform a momentum-shell RG [13] on model (3) integrating out all modes with wave vectors $\Lambda e^{-d l}<k \leq \Lambda$ of both fields $\phi$ and $\tilde{\phi}$. This calculation is performed only to first order in $\gamma$ without restricting its functional form. In order to restore the original value of the cutoff, we then rescale $\mathbf{r} \rightarrow e^{d l} \mathbf{r}$, and simultaneously $t \rightarrow e^{2 d l} t, \tilde{\phi}_{l} \rightarrow e^{-2 d l} \tilde{\phi}_{l+d l}$, and $\phi_{l} \rightarrow \phi_{l+d l}$ according to the scale invariance in absence of pinning.

This procedure easily gives the flow equation for the correlator

$$
\partial_{l} \gamma_{l}(\mathbf{r})=\left(4+\mathbf{r} \cdot \nabla-2 n_{l}\right) \gamma_{l}(\mathbf{r})
$$


which is exact in this order. On the same level, there is no renormalization of $\mu, \vartheta$, and $\kappa$. However, new interaction terms are generated, which are not present in the unrenormalized action. In order to keep the theory simple, i.e. the number of parameters small, we replace these terms approximately by contributions to the flow of $\mu, \vartheta$, and $\kappa$. Following Nozières and Gallet [2], we thereby take the coupling between the smooth modes (small $k$ ) into account in order to avoid artifacts related to the special choice of the cutoff procedure. The resulting flow equations are $(\boldsymbol{\rho}=\Lambda \mathbf{r})$

$$
\begin{aligned}
\partial_{l} \kappa_{l} & =\frac{1}{8 \pi \kappa_{l} \Lambda^{4}} \int d^{2} \rho \rho^{2} \gamma_{l}(\boldsymbol{\rho} / \Lambda) A\left(n_{l}, \rho\right) \\
\partial_{l} \vartheta_{l} & =\frac{\vartheta_{l}}{2 \pi \kappa_{l}^{2} \Lambda^{4}} \int d^{2} \rho \gamma_{l}(\boldsymbol{\rho} / \Lambda) B\left(n_{l}, \rho\right) \\
\partial_{l} \mu_{l} & =-\frac{\mu_{l}}{\vartheta_{l}} \partial_{l} \vartheta_{l} \\
\partial_{l} \tilde{J}_{l} & =2 \tilde{J}_{l}
\end{aligned}
$$

with the definitions

$$
\begin{aligned}
n_{l} & :=\frac{\mu_{l} \vartheta_{l}}{8 \pi \kappa_{l}} \\
A(n, \rho) & :=\mathrm{J}_{0}(\rho) e^{-n \hat{C}^{(0)}(0, \rho)} \\
B(n, \rho) & :=\mathrm{J}_{0}(\rho) \int_{0}^{\infty} d \tau e^{-\tau-n \hat{C}^{(0)}(\tau, \rho)} .
\end{aligned}
$$

Under renormalization the effective stiffness of the interface is increased, $\partial_{l} \kappa_{l}>0$, and the roughness amplitude is decreased, $\partial_{l} n_{l}=-\left(n_{l} / \kappa_{l}\right) \partial_{l} \kappa_{l}<0$. As long as disorder is renormalized to zero, it will have the effect to reduce the equal-time roughness given by $n_{\infty}$, since $C(t, \mathbf{r}) \approx n_{\infty} \hat{C}^{(0)}\left(\mu_{\infty} \kappa_{\infty} \Lambda^{2} t, \Lambda r\right)$. The invariance $\partial_{l}\left(\mu_{l} \vartheta_{l}\right)=0$ expresses the preservation of the fluctuation-dissipation theorem under renormalization. This allows a stepwise solution of the flow equations: first for $\gamma$ and $\kappa$, since their flow equations are closed. These quantities completely determine the static properties of the system. Afterwards one can solve for $\vartheta$ or $\mu$, which affect only the dynamics. Pinning always reduces mobility, $\partial_{l} \mu_{l}<0$, even if disorder is renormalized to zero on large length scales. The driving force leads to a drift velocity $\langle\dot{\phi}\rangle \approx \mu_{l^{*}} m F$. In the driven case renormalization has to be stopped at a value $l^{*}$ of 
the flow parameter determined by $m F=e^{-2 l^{*}} \kappa_{l^{*}} \Lambda^{2}$ since a finite drift velocity washes out the effect of pinning.

Now we discuss the phase boundary separating the regions, where disorder changes from being irrelevant to being relevant. The flow equation for $\gamma$ can directly be integrated:

$$
\gamma_{l}(\mathbf{r})=\exp \left[-2 \int_{0}^{l} d l^{\prime}\left(n_{l^{\prime}}-2\right)\right] \gamma_{0}\left(e^{l} \mathbf{r}\right) .
$$

In two extreme cases the relevance of disorder is obvious. The first case $\gamma_{0}(\mathbf{r})=g_{0}$ represents perfect crystal planes. From eq. (90) we find the boundary at $n_{\infty}=2$ which is the usual roughening transition. In the limit $\chi \rightarrow 0$ of a weak potential the transition temperature is $T_{\mathrm{R}}^{(0)}=8 \pi K$. For finite $\chi$ the transition temperature $T_{\mathrm{R}}$ will be higher than $T_{\mathrm{R}}^{(0)}$, since $n_{\infty}<n_{0}$. In the second case $\gamma_{0}(\mathbf{r})=g_{0} \delta(\Lambda \mathbf{r})$ of extremely deformed crystal planes we find the boundary at $n_{\infty}=1$. The superroughening transition occurs at a temperature $T=T_{\mathrm{SR}}=4 \pi K$ independently of disorder strength, since here always $n_{\infty}=n_{0}$. This universality of the transition temperature is special for a infinitely sharp $\gamma_{0}(\mathbf{r})$. A finite width leads to $\partial_{l} n_{l}<0$ and hence an increased transition temperature $T_{\mathrm{SR}}>4 \pi K$. Such a shift has been observed recently in simulations of superroughening [14]. In a comparison with such data, the lateral lattice spacing should be taken as width.

The decisive interpolation between theses cases of very broad and very sharp $\gamma(\mathbf{r})$ is provided when $\gamma_{0}(\boldsymbol{\rho} / \Lambda) \approx c_{0} \rho^{-2 \alpha}$ for large $r$ with some amplitude $c_{0}>0$ and some exponent $\alpha \geq 0$. This means, that the crystal planes themselves have logarithmic roughness. Renormalization preserves the asymptotic exponent $\alpha$, but the amplitude is changed: $c_{l} \propto \exp \left[-2 \int_{0}^{l} d l^{\prime}\left(n_{l^{\prime}}+\alpha-2\right)\right] c_{0}$. Thus we expect pinning to become relevant for the largescale behavior of the interface at $n_{\infty}=2-\alpha$.

To be more specific, we solve the flow equations (7), after eq. (9) has been plugged in. For weak pinning, an eventual transition is related to large values of the flow parameter, where the flow equations simplify:

$$
\partial_{l} n_{l}=-y_{l}^{2}
$$




$$
\begin{array}{r}
y_{l}^{2} \approx \begin{cases}A^{<} e^{-2 \int_{0}^{l} d l^{\prime}\left(n_{l^{\prime}}+\alpha-2\right)} & \text { for } \alpha<2 \\
A^{>} e^{-2 \int_{0}^{l} d l^{\prime} n_{l^{\prime}}} & \text { for } \alpha>2\end{cases} \\
\partial_{l} \ln \vartheta_{l} \approx \begin{cases}B^{<} e^{-2 \int_{0}^{l} d l^{\prime}\left(n_{l^{\prime}}+\alpha-2\right)} & \text { for } \alpha<1 \\
B^{>} e^{-2 \int_{0}^{l} d l^{\prime}\left(n_{l^{\prime}}-1\right)} & \text { for } \alpha>1 .\end{cases}
\end{array}
$$

The various coefficients like $A^{<}$follow from the original equations (7) using suitable approximations for large $l$, which are not displayed here since they are clumsy and straightforward [15]. These coefficients are positive and well-defined at least for $n>5 / 4-\alpha$. This region includes the transitions under discussion. As long as we are not too close to that border, we may ignore the implicit dependence of the coefficients on $n$. Due to the ambivalent asymptotic behavior of flow equations (10) one has to distinguish three regimes depending on $\alpha$ displayed in Fig. 2.

Regime $0 \leq \alpha<1$. The flow trajectories in the $(n, y)$-plane have hyperbolic structure $\left(n_{l}+\alpha-2\right)^{2}-y_{l}^{2}=$ const. like in the Kosterlitz-Thouless transition [16]. In addition, $\vartheta_{l}=\vartheta_{0} \exp \left[-\left(A^{<} / B^{<}\right)\left(n_{l}-n_{0}\right)\right]$. Disorder is irrelevant as long as $n_{0}+\alpha-2>y_{0}$ since then $y_{\infty}=0$ and $n_{\infty}>2-\alpha$. For weak disorder strength, the boundary $n_{\infty}=2-\alpha$ corresponds to a temperature $T_{\mathrm{R}}(\alpha) \gtrsim(2-\alpha) 4 \pi K$. Approaching the transition, $n_{\infty}$ and $\vartheta_{\infty}$ remain finite, as well as the effective mobility $\mu_{\infty}=\mu_{0} \vartheta_{0} / \vartheta_{\infty}$. Thus for $0 \leq \alpha<1$ the transition is qualitatively similar to the roughening case $\alpha=0$. In the low-temperature range, the perturbative flow equations loose their validity, since the strength of pinning increases. However, as the effective amplitude $c_{l}$ of $\gamma_{l}$ diverges as discussed above, we expect the interface to lock into the pinning potential. This implies, that the equal-time roughness is $C(0, \mathbf{r}) \sim \alpha \ln \left(\Lambda^{2} r^{2}\right)$ below the transition, whereas it is $C(0, \mathbf{r}) \sim n_{\infty} \ln \left(\Lambda^{2} r^{2}\right)$ above. Hence the transition is discontinuous. Following $\alpha \rightarrow 1^{-}$along the transition, the magnitude of the jump disappears, since for $\alpha=n_{\infty}$ the logarithmic roughness of the crystal planes and interface coincide. In a similar way the (linear) mobility behaves discontinuously at the transition since it remains finite approaching the transition from above and it is zero for the locked-in interface. The jump of the roughness at the transition from $n_{\infty}=2-\alpha$ to $\alpha$ is 
universal, whereas the jump of mobility depends on all parameters.

Proceeding to the regime $1<\alpha<2$, only the flow equation for $\vartheta$ changes. It exhibits now a continuous divergence, if $n_{\infty}$ approaches the value 1 from above, where $T_{\mathrm{SR}}(\alpha) \gtrsim 4 \pi K$. Thus the interface becomes immobile $\left(\mu_{\infty}=0\right)$ at that point, although it does not lock in, what happens only at the lower temperature $T_{\mathrm{R}}(\alpha)$ given before. Thus our flow equations suggest a sequence of two transitions: a dynamic one and a static one. However, due to their perturbative nature, we are not really in position to characterize the low-temperature phase $n<1$. Possibly the lock-in transition for $1<\alpha<2$ might be an artifact of the first order calculation. If the transition exists, the interface is rougher below the transition than above.

In the third regime $2<\alpha$ we also have to use the second flow equation of $n$. Nevertheless, the flow remains hyperbolic with $n_{l}^{2}-y_{l}^{2}=$ const and every $n_{0}>0$ is renormalized only by a small amount. Thus disorder is always irrelevant for $n$, a lock-in transition becomes impossible. The dynamic transition is the same as in the regime $1<\alpha<2$. Again, we can not seriously say something for $n<1$, except that the linear mobility should continue to be zero. The controversy mentioned in the introduction is precisely about this phase, since the commonly used $\gamma(\mathbf{r})$ decaying faster than any power belong to $\alpha=\infty$. However, there is agreement that below the superroughening transition the interface is at least as rough as at the transition. This fact is not captured by the first order flow equations.

To sum up, we have performed a dynamical RG for a disordered sine-Gordon model in the presence of weak pinning with arbitrary disorder correlations. Thereby roughening and superroughening transition could be merged for a correlation $\gamma_{0}(\mathbf{r}) \sim r^{-2 \alpha}$. Disorder is irrelevant for the interface at temperatures $T \gtrsim \max (2-\alpha, 1) 4 \pi K$. In the range $0 \leq \alpha<1$ the transition is of roughening type with a discontinuous jump of roughness and mobility. The jump of roughness is universal. This discontinuity decreases, as $\alpha=1$ is approached. For $\alpha>1$ the transition is of the continuous superroughening type. The transition temperature is non-universal in all regimes, as long as disorder correlations have a finite range.

A similar interpolation between transitions has been found in a study of vortex glasses [8] 
with anisotropic disorder correlations, which are able to merge the vortex-glass transition (equivalent to superroughening) and the Bose-glass transition for $\gamma(x, y)=g \delta(\Lambda x)$. The latter was found at $n=3$, as is immediately reproduced by our functional flow equation (6). In fact, this equation holds also in the anisotropic case as long as the anisotropy of $\kappa$ can be neglected.

The author gratefully acknowledges helpful discussions with J. Krug, T. Nattermann, and L.-H. Tang. 


\section{REFERENCES}

[1] H. van Beijeren and I. Nolden, in Structure and Dynamics of Surfaces II, edited by W. Schommers and P. von Blanckenhagen (Springer, Berlin 1987).

[2] P. Nozières and F. Gallet, J. de Physique 48, 353 (1987); see also P. Nozières in "Solids far from equilibrium", Ed. C. Godrèche, Cambridge Univ. Press (1992), p. 1.

[3] J. Toner and D.P. DiVincenzo, Phys. Rev. B 41, 632 (1990).

[4] J.L. Cardy and S. Ostlund, Phys. Rev. B 28, 6899 (1982); Y.Y. Goldschmidt and B. Schaub, Nucl. Phys. B251, 77 (1985).

[5] Y.-C. Tsai and Y. Shapir, Phys. Rev. Lett. 69, 1773 (1992); Phys. Rev. B 50, 3546 (1994).

[6] This is the case if the external field has $p$-fold symmetry breaking and $p$ is sufficiently large [4].

[7] M.P.A. Fisher, Phys. Rev. Lett. 62, 1415 (1989); T. Nattermann, Phys. Rev. Lett. 64, 2454 (1990); T. Nattermann, I. Lyuksyutov, and M. Schwarz, Europhys. Lett. 16, 295 (1991).

[8] T. Hwa, D.R. Nelson, and V.M. Vinokur, Phys. Rev. B 48, 1167 (1993).

[9] S.E. Korshunov, Phys. Rev. B 48, 3969 (1993); T. Giamarchi and P. LeDoussal, Phys. Rev. Lett. 71, 1530 (1994).

[10] G.G. Batrouni and T. Hwa, Phys. Rev. Lett. 72, 4133 (1994); D. Cule and Y. Shapir, Phys. Rev. Lett. 74, 114 (1995).

[11] Since $d$ is a continuous function, one can uniquely follow its profile when $\mathbf{r}$ is changed and height differences are single-valued. This is in contrast to laterally dicrete, where height differences can arbitrarily be shifted by integer multiples of $2 \pi$. For a comparision with lattice models one should refer to $\gamma_{0}$ rather than to $\Delta$. 
[12] P.C. Martin, E.D. Siggia, and H.A. Rose, Phys. Rev. A 8, 423 (1973).

[13] F.J. Wegner and A. Houghton, Phys. Rev. A 8, 401 (1973); see also T.S. Chang, D.D. Vvedensky, and J.F. Nicoll, Phys. Reports 217, 279 (1992).

[14] D. Cule, preprint cond-mat 9505130 (1995).

[15] Remarkably, for $\gamma_{0}(\mathbf{r})=g_{0}$ the flow equations of $g$ and $\kappa$ exactly agree with the static ones in Ref. [2]. The dynamical flow equations therein are different, since only modes of $\phi$ but not of $\tilde{\phi}$ have been integrated out. For $\gamma_{0}(\mathbf{r})=4 \pi g_{0} \delta(\Lambda \mathbf{r})$ the flow of $\vartheta$ or $\mu$ is by a factor $\approx 10$ smaller than the first-order contributions in Ref. [5].

[16] J.M. Kosterlitz and D. Thouless, J. Phys. C 6, 1181 (1973); J.M. Kosterlitz, J. Phys. C 7, 1046 (1974). 


\section{FIGURES}

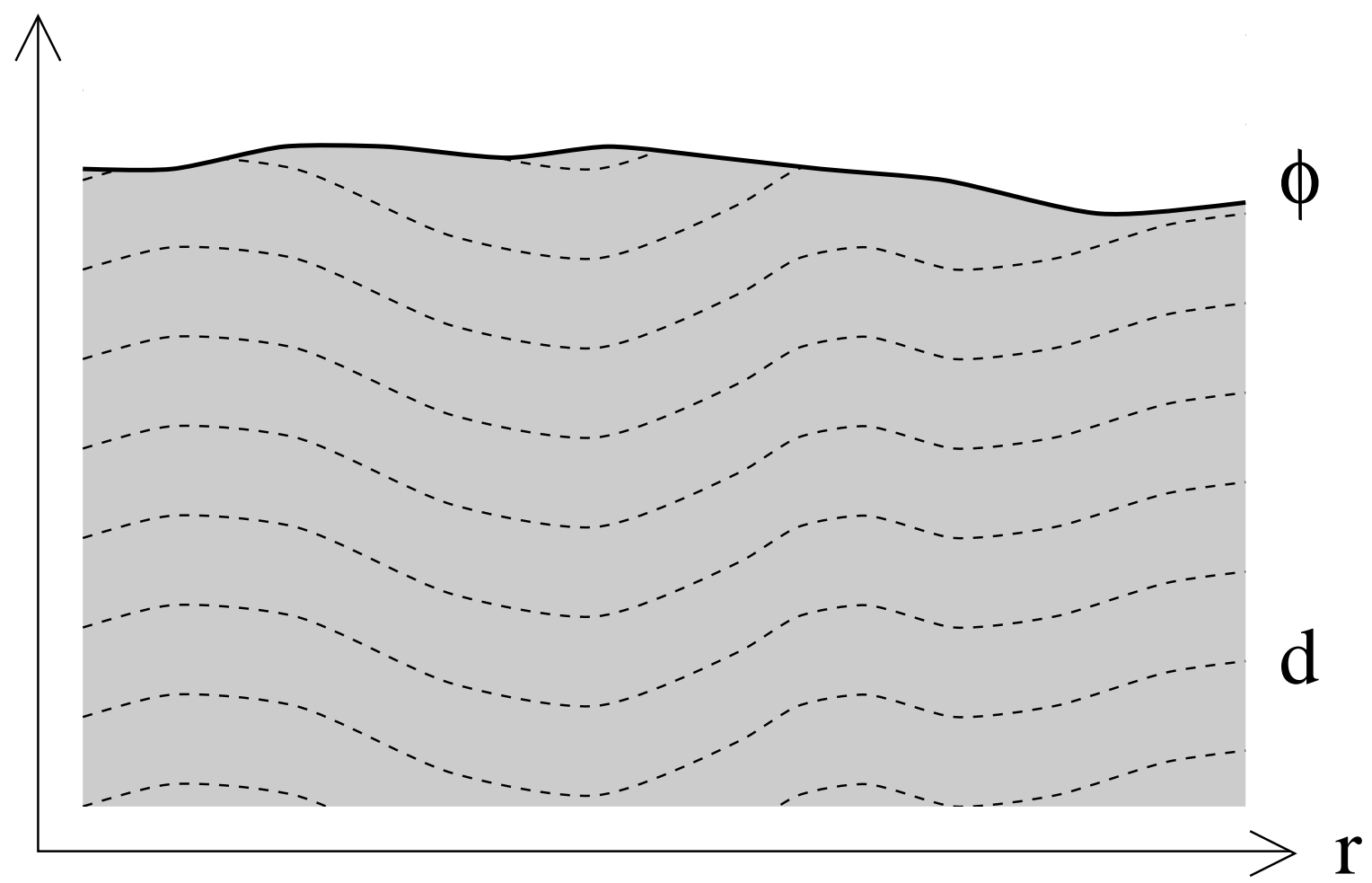

FIG. 1. Schematic sketch of a crystal (hatched area) with interface $\phi$ (bold line) and disordered crystal planes (dashed lines) parameterized by $d$. 


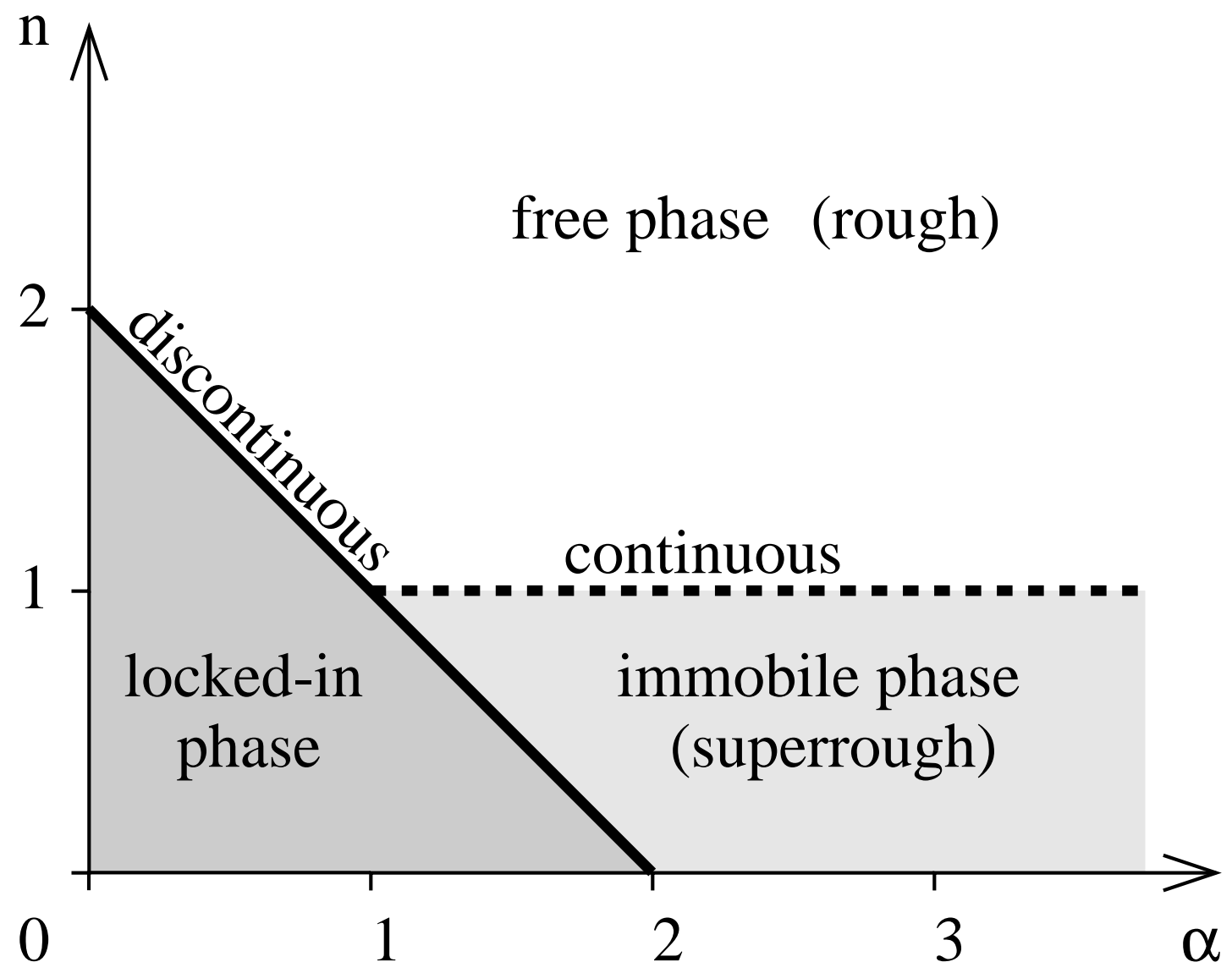

FIG. 2. Phase-diagram: The free phase, where pinning is irrelevant is bound by $T /(4 \pi K) \approx n=\max (2-\alpha, 1)$. For a range of disorder correlations $\alpha \geq 0$ a discontinuous lock-in transition occurs, whereas for $\alpha>1$ a continuous superroughening transition takes place. 